Paper No. W.1.2

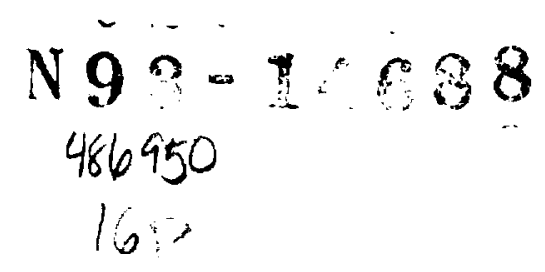

RECENT ADVANCES IN NUMERICAL SIMULATION AND CONTROL OF ASYMMETRIC FLOWS AROUND SLENDER BODIES

O. A. Kandil, T.-C. Wong, H. H. Sharaf

Old Dominion University, Norfolk, VA, USA

C. H. Liu

NASA Langley Research Center, Hampton, VA, USA

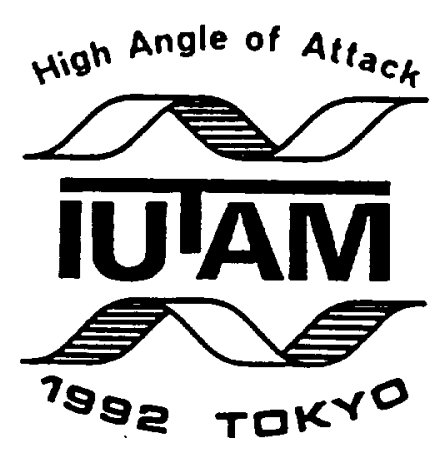

IUTAM SYMPOSIUM ON FLUID

DYNAMICS OF HIGH ANGLE OF ATTACK

Tokyo, Japan - September 13-17, 1992 



\title{
RECENT ADVANCES IN NUMERICAL SIMULATION AND CONTROL OF ASYMMETRIC FLOWS AROUND SLENDER BODIES
}

\author{
O. A. Kandil. T.-C. Wong, H. H. Sharaf El-Din \\ Dept. of Mechanical Engineering and Mechanics \\ Old Dominion University, Norfolk, VA \\ C. H. Liu \\ Theoretical Flow Physics Br. \\ NASA Langley, Hampton, VA
}

\section{Summary}

The problems of asymmetric flow around slender bodies and its control are formulated using the unsteady, compressible, thin-layer or full Navier-Stokes equations which are solved using an implicit, flux-difference splitting, finite-volume scheme. The problem is numerically simulated for both locally-conical and three-dimensional flows. The numerical applications include studies of the effects of relative incidence, Mach number and Reynolds number on the flow asymmetry. For the control of flow asymmetry, the numerical simulation cover passive and active control methods. For the passive control, the effectiveness of vertical fins placed in the leeward plane of geometric symmetry and side strakes with different orientations is studied. For the active control, the effectiveness of normal and tangential flow injection and surface heating and a combination of these methods is studied.

\section{Introduction}

Flow asymmetry around pointed slender cones develops at critical values of relative incidence (ratio of angle of attack to nose semiapex angle) due to short-duration transient disturbances or forced disturbances. The origin of the transient disturbances may be a transient side slip, an acoustic disturbance, or similar disturbances of short duration. The origin of forced disturbances is geometric imperfections in the nose or similar disturbances of permanent nature. Flow asymmetry produces side forces, asymmetric lifting forces and corresponding yawing, rolling and pitching moments that might be larger than those available by the control system of the vehicle. Currently, research efforts are devoted for eliminating or alleviating flow asymmetry and its corresponding asymmetric loads. Various methods of passive and active control are being studied to learn about their control effectiveness.

In several recent papers by Kandil, et al. [1]-[4], the unsteady, thin-layer, compressible Navier-Stokes equations have been used to simulate steady and unsteady, asymmetric vortex flows, including their passive control, around cones with different cross-sectional shapes. The emphasis of these papers was extensive computational studies of the parameters which influence the asymmetric flow phenomenon and its passive control. Since the computational cost associated with the solution of three-dimensional-flow problems at reasonable flow resolution is very expensive, all the computational solutions were obtained using a locallyconical flow assumption. Such an assumption reduces the problem solution to that on 
two conical planes, which are in close proximity of each other, and hence it reduces the computational cost by an order of magnitude. Moreover, such solutions still provide extensive understanding of the flow physics since one can use very fine grids for reasonable flow resolution.

In a later paper, by Kandil, et al. [5], the full Navier-Stokes solutions were compared with the thin-layer Navier-Stokes solutions. It was shown that the full Navier-Stokes solutions produced thicker free-shear layers and more vortex-core resolution as compared with those of the thin-layer Navier-Stokes equations. In reference [5], a few tentative three-dimensional flow solutions were also presented.

Substantial research efforts have recently been devoted for eliminating or alleviating flow asymmetry and its corresponding side force. In the experimental area, several passive-control methods [6]-[8] and active-control methods [9]-[13] have been investigated. Computational simulations have also been used to investigate the effectiveness of several passive-control methods [1]-[5] and active-control methods [12], [14], [15]. Various methods of passive control were demonstrated in the above references which include the use of vertical fins along the leeward plane of geometric symmetry, thin and thick side strakes with different orientations, and rotatable forebody tips which have variable cross section (from a circular shape at its base to an elliptic shape at its tip). It was shown by Kandil, et al. [4] that side-strakes control is more practical than the vertical-fin control since the former was more effective over a wide range of angle of attack than the former. Moreover, side-strake control provided an additional lifting force. However, the effectiveness of the side-strake control terminates at very high angles of attack for the considered strake geometry and flow conditions.

Various active-control methods have been used which include forebody blowing and movable forebody strakes. The forebody blowing methods include forward blowing, normal blowing, aft blowing and tangential blowing. The main concept of forebody blowing is to control flow separation on the forebody and to create yawing forces and moments which can be utilized in controlling the body.

In this paper, we present samples of simulating asymmetric locally-conical and threedimensional flows around cones. Next, we present samples of simulation for passive control using a vertical fin and a side strake. Samples of simulating active control using normal and tangential flow injection, surface heating and hybrid methods are also presented.

\section{Highlights for Formulation and Computational Schemes}

Formulation: The asymmetric-flow problems including their passive and active controls are formulated using the conservative form of the unsteady, compressible, thin or full Navier- 
Stokes equations in terms of time-independent, body-conforming coordinates. The equations are given in Ref. [5] and hence, they are not repeated here.

The boundary and initial conditions vary according to the problem under consideration. The boundary conditions are explicitly satisfied. In general, they include inflow-outflow conditions and solid-boundary conditions. For problems of flow asymmetry, where the flow is solved throughout the whole computational domain, periodic boundary conditions are used at the plane of geometric symmetry of the problem.

For the asymmetric flow problems around slender bodies and for supersonic inflow-outflow boundary, the Riemann-invariant boundary conditions are used. They require that the inflow variables be at the freestream conditions, and the conical shock enclosing the body be captured as part of the solution. For supersonic outflow boundary, the Riemann-invariant boundary conditions require that all flow variables be extrapolated from the interior cells. On the solid boundary, without injection or heating, the no-slip and no-penetration conditions are enforced. Moreover, the zero normal-pressure gradient and adiabatic boundary conditions are enforced. For the active control problems, the mass-flow rate is specified at the body surface for the normal injection control and the temperature distribution is specified at the surface for the heating control. For the tangential flow injection, the mass flow rate and velocity profile are specified at the lip exit.

The initial conditions correspond to the uniform flow conditions with $u_{1}=u_{2}=u_{3}=0$ on the solid boundary. These conditions are used to obtain the asymmetric flow solution. Next, the flow control conditions are enforced and the previously obtained asymmetric solution is used for the initial conditions of the active control problem.

Computational Scheme: The implicit, upwind, flux-difference splitting, finite-volume scheme is used to solve the unsteady, compressible, full Navier-Stokes equations. The scheme uses the flux-difference splitting scheme of Roe which is based on the solution of the approximate Riemann problem. In the Roe scheme, the inviscid flux difference at the interface of computational cells is split into two parts; left and right flux differences. The splitting is accomplished according to the signs of the eigenvalues of the Roe averaged-Jacobian matrix of the inviscid fluxes at the cell interface. The smooth flux limiter is used to eliminate oscillations at locations of large flow gradients. The viscous-and heat-flux terms are linearized and the cross-derivative terms are eliminated in the implicit operator. The viscous terms are differenced using a second-order accurate central differencing. The resulting difference equation is approximately factored and is solved in three sweeps in the $\xi^{1}, \xi^{2}$, and $\xi^{3}$ directions. The computational scheme is coded in the computer program "FTNS3D".

For the locally-conical flow solutions, an axial station of $x_{1}=1.0$ is selected and the components of the flowfield vector are forced to be equal between this axial station and 
another axial station in close proximity to $x_{1}=1.0$. This ensures that the flow variables are locally independent of the axial direction at $x_{1}=1.0$ (Kandil, et al. [5]).

\section{Computational Applications and Discussion}

Asymmetric Steady Flow (locally-conical solution): Figure 1 shows the residual error versus the number of iterations, surface-pressure (SP) coefficient, cross-flow velocity and total-pressure-loss (TPL) contour for the solution around a $5^{\circ}$-semiapex circular cone. Two computer codes (CFL3D and ICF3D) which solve the thin-layer Navier-Stokes equations are used to validate the asymmetric flow solution. The logarithmic-residual-error curve shows the stages through which the solution goes until a stable asymmetric steady solution is obtained. A grid of $161 \times 81 \times 2$ points in the wrap-around, normal and axial directions, respectively, with minimum spacing of $10^{-4}$, has been used. The computational domain extends around the body to $21 \mathrm{r}$ where $\mathrm{r}$ is the local radius of the cone. For these critical conditions, the asymmetry is developed due to random disturbances; such as the machine round-off-error for the CFL3D solution.

Asymmetric Unsteady Flow (locally-conical solution): Figure 2 shows the results for the solution of the flow around the same cone using the same grid, where $\alpha=30^{\circ}$. Here, the solution is validated by using the thin-layer and full, Navier-Stokes equations using the flux-difference splitting (FDS) scheme and the flux-vector splitting (FVS) scheme. All the solutions show asymmetric, periodic flow with vortex shedding. The figures show snapshots of TPL contours over a half cycle of periodic response. The periodicity is substantiated by the first and last snapshot in each row. It is clearly observed that they are mirror image of each other over the half cycle.

Asymmetric Steady Flow (three-dimensional solutions): Figures 3 and 4 show the asymmetric flow results for the flows around a $5^{\circ}$-semiapex cone and a $5^{\circ}$-semiapex cone with cylindrical after-body configuration. A grid of $161 \times 81 \times 65$ points in the wrap-around, normal and axial directions, respectively, with minimum spacing of $10^{-6}$ has been used. For the cone solution, the spatial flow asymmetry is qualitatively similar to that of the temporal flow asymmetry of the locally-conical flow solution of Fig. 2. For the cone-cylinder configuration at the same angle of attack and Mach number as those of the cone case, it is concluded that the cylindrical afterbody enhances the flow asymmetry at lower Reynolds number. These solutions are obtained using the thin-layer Navier-Stokes equations.

Passive Control Using Fins and Strakes (locally-conical solutions): Figures 5 and 6 show the solutions using passive flow controls through a vertical fin placed in the leeward plane of geometric symmetry (Fig. 5) or side strakes (Fig. 6). It is concluded that the fin height, $h$, must be at least equal to or greater than the height of the free-shear layers in order 
to yield a symmetric flow. The side-strake control is more practical than the fin control since it is more effective for high angles of attack than the fin, and moreover it provides additional lifting force. These solutions are obtained using the thin-layer Navier-Stokes equations.

Active-Control Using Normal Flow Injection: Figure 7 shows the history of the locallyconicaly full Navier-Stokes solutions for active control around a $5^{\circ}$-semiapex cone. The control is achieved by injecting flow from circumferential ports in the circumferential angle range of $\theta= \pm 67.5^{\circ}$. A variable mass-flow-rate injection of maximum rate of 0.03 is used. The mass flow rate is proportional to the difference in the surface pressure between the left and right sides of the cone. Figure 8 shows the effectiveness of this flow injection as the angle of attack is increased up to $30^{\circ}$, where flow asymmetry develops again. The solution is obtained using the full Navier-Stokes solver, FTNS-3D code, on a grid of $241 \times 81 \times 2$.

Hybrid Active Control Using Heating and Injection: Since normal flow injection failed to yield asymmetric solutions at angles of attack as high as $30^{\circ}$, hybrid methods of active control are investigated. Figure 9 shows the effectiveness of hybrid surface heating and variable mass flow normal injection for the same cone as the angle of attack is increased. It is seen that this method is very promising at high angles of attack. Here, symmetric surface pressure and not symmetric flow is obtained at $\alpha=38^{\circ}$ and $42^{\circ}$. The surface temperature is taken as $T_{s}=5 T_{\infty}$ and the maximum mass flow rate is 0.05 . The solution is obtained using the full Navier-Stokes solver, FTNS-3D code, on the same grid as that of Fig. 8.

Active Control Using Tangential Flow Injection: Figure 10 shows the results for active control using injection of flow from side lips. The radius of the lower portion is 1.05 that of the upper portion. The maximum mass flow rate is 0.2 and a parabolic velocity profile is assumed at the lip exit. The solution is obtained by using the full Navier-Stokes solver, FTNS-3D, code and a multi-block scheme to grid the lip-flow exit and the upper and lower flow regions. It is seen that this method is effective up to $30^{\circ}$ angle of attack.

\section{Concluding Remarks}

The unsteady, compressible, thin-layer and full Navier-Stokes equations have been used to solve for asymmetric steady and unsteady, locally-conical and three-dimensional flows around circular cones at high angles of attack. Passive and active control methods have been applied to study their effectiveness to yield either a symmetric flow or a symmetric surface pressure distribution and hence removing the side forces. Work is underway to use other active control methods as well as hybrid passive-active control methods. 


\section{Acknowledgement}

For the first three authors, this research work has been supported by the NASA Langley Research Center under Grant No. NAG-1-994. The computational resources provided by the NASA Langley Research are appreciated.

\section{References}

1. Kandil, O. A.; Wong, T-C.; Liu, C. H.: Prediction of Steady and Unsteady Asymmetric Vortical Flow Around Cones. ALAA 90-0598, 1990. Also in AIAA Journal, Vol. 29. No. 12, pp. 1269-1278, 1991.

2. Kandil, O. A.; Wong, T-C.; Liu, C. H.: Asymmetric Flow Around Cones with Noncircular Sections. AGARD Symposium on Missile Aerodynamics, AGARD CP No. 493, Friedrickshafen, Germany, pp. 16.1-16.11, 1990.

3. Kandil, O. A.; Wong, T-C.; Liu, C. H.: Numerical Simulation of Steady and Unsteady Asymmetric Vortical Flows. ASME Symposium on Non-Steady Fluid Dynamics, FEDVol. 92, Toronto, Canada, pp. 99-108, 1990. Also in the Journal of Fluids and Structures, Vol. 6, pp. 249-265, 1992.

4. Kandil, O. A;, Wong, T-C.; Kandil, H., A.; Liu, C. H.: Computation and Control of Asymmetric Vortex Flow Around Circular Cones Using Navier-Stokes Equations. ICAS Paper No. 3.5.3, Vol. 2, Stockholm, Sweden, pp. 883-893, 1990.

5. Kandil, O. A.; Wong, T-C.; Kandil, H. A.; Liu, C. H.: Thin-Layer and Full Navier-Stokes, Locally-Conical and Three-Dimensional Asymmetric Solutions. AIAA 91-0547, 1991.

6. Stahl, W.: Suppression of Asymmetry of Vortex Flow Behind a Circular Cone at High Incidence. Proceedings of the AIAA Atmospheric Flight Mechanics Conference, Boston, MA, ALAA 39-3372-CP, pp. 231-236, 1989.

7. Ng, T. T.: On Leading Edge Vortex and Its Control. Proceedings of the AIAA Atmospheric Flight Mechanics Conference, Boston, MA, AIAA 89-3346-CP, pp. 1-15, 1989.

8. Moskovitz, C., Hall, R.: DeJarnette; Experimental Investigation of a New Device to Control the Asymmetric Flowfield on Forebodies at Large Angles of Attack. ALAA 90-0069, 1990.

9. Skow, A. M.; Peake, D. J.: Control of the Forebody Vortex Orientation by Asymmetric Air Injection, (Part B) - Details of the Flow Structure. AGARD-LS-121, High Angleof-Attack Aerodynamics, pp. 10.1-10.22, 1982.

10. Ng, T. T.; Suarez, C. J.: Malcolm, N.; Forebody Vortex Control Using Slot Blowing. Proceedings of the AIAA 9th Applied Aerodynamics Conference, Baltimore, Maryland, AIAA 91-3254-CP, pp. 412-421, 1991.

11. Gittner, M. N.; Chokani, N.: An Experimental Study of the Effects of Aft Blowing on a 3.0 Caliber Tangent Ogive Body at High Angles of Attack. Proceedings of the AIAA 9th Applied Aerodynamics Conference, Baltimore, Maryland, AIAA 91-3252-CP, pp. 390-399, 1991. 
12. Font, G. I.; Celik, Z. Z.; Roberts, L.: A Numerical and Experimental Study of Tangential Jet Blowing Applied to Bodies at High Angles of Attack. Proceedings of the AIAA 9th Applied Aerodynamics Conference, Baltimore, Maryland, AIAA 91-3253-CP, pp. 400-411, 1991.

13. Ng, T. T.: Aerodynamic Control of NASP-Type Vehicles Through Vortex Manipulation. AIAA 90-0594, 1990.

14. Tavella, D. A.; Schiff, L. B.: Cummings, R. M.; Pneumatic Vortical Flow Control at High Angles of Attack. AIAA 90-0098, 1990.

15. Gee, K.; Tavella, D.; Schiff, L. S.: Comptutational Optimization of a Pneumatic Forebody Flow Control Concept. Proceedings of the AIAA 9th Applied Aerodynamics Conference, Baltimore, Maryland, AIAA 91-3249-CP, pp. 370-380, 1991. 


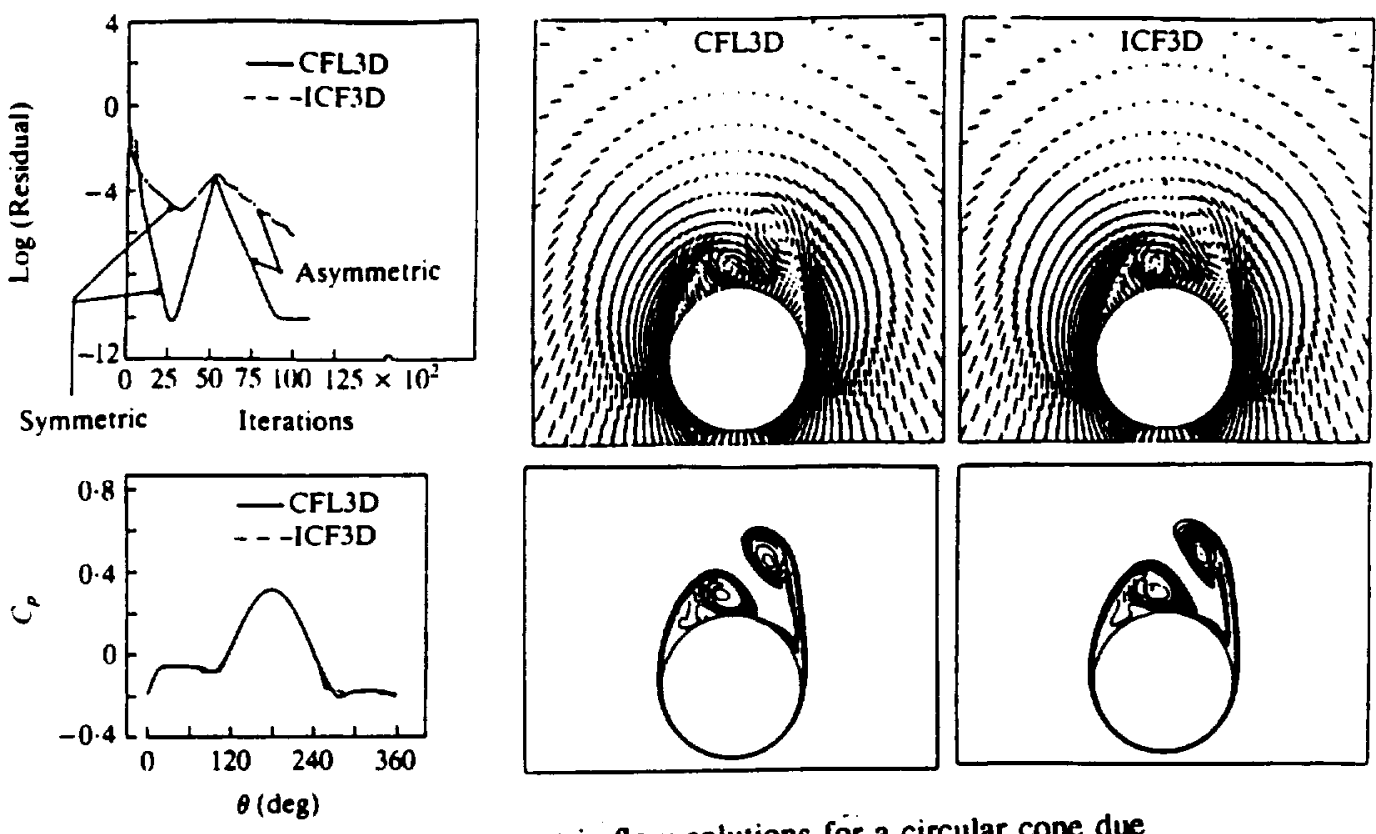

Figure 1. Steady asymmetric flow solutions for a circular cone due to random disturbances, $a=20 \mathrm{deg}, M_{\infty}=1.8, R_{e}=10^{5}$

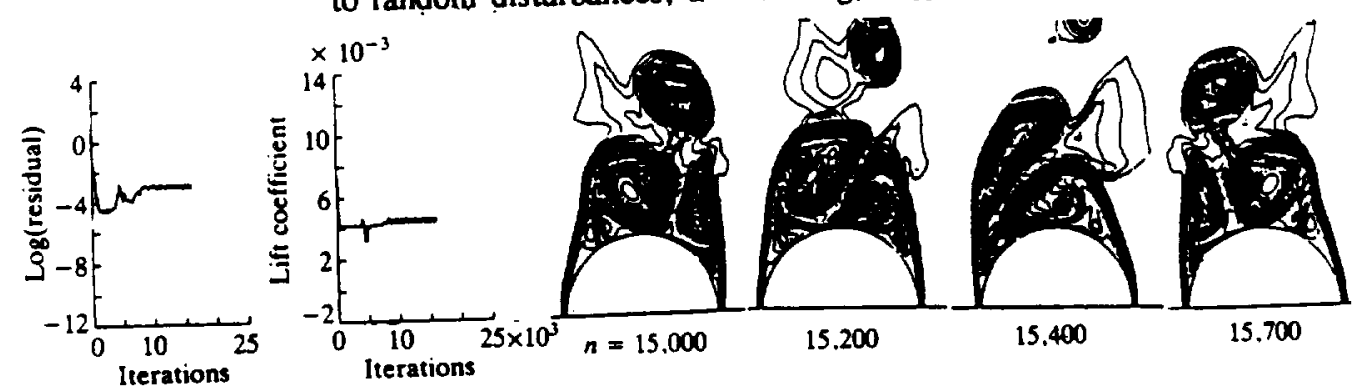

Thin-layer eqs. (FDS)
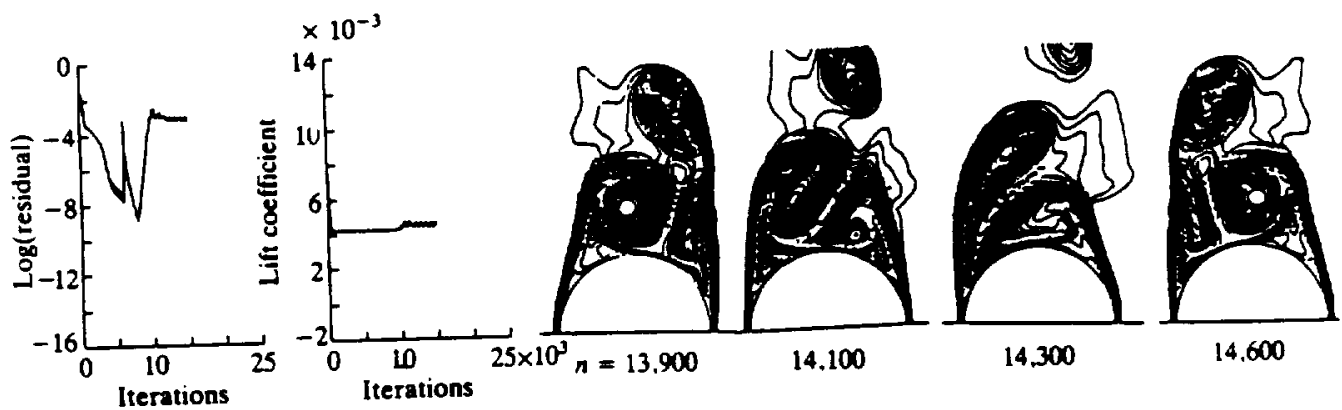

Thin-layer eqs. (FVS)
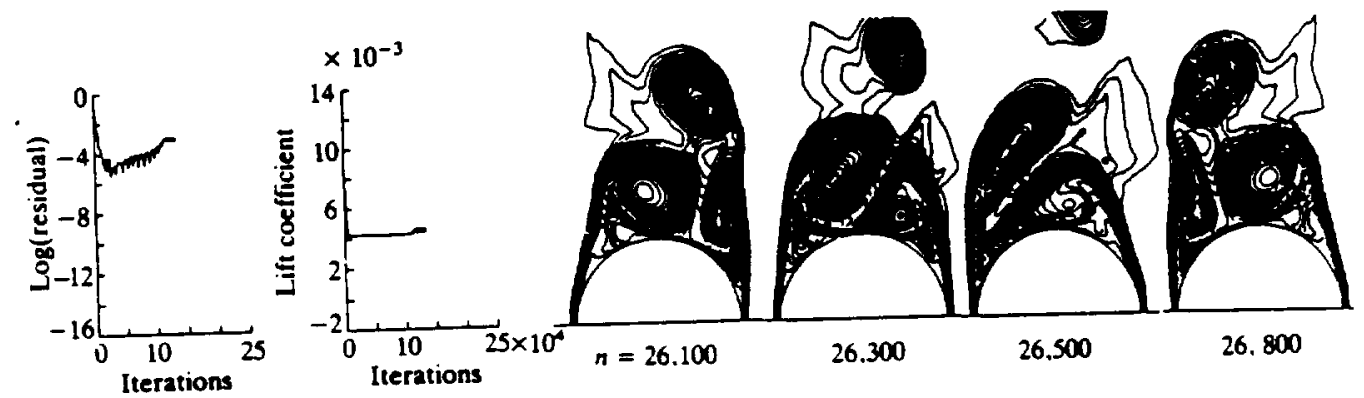

Full Navier-Stokes eqs. (FDS)

Figure 2. Comparison of unsteady asymmetric now solutions with vortex shedding around a circular cone; periodic flow response; $\alpha=30^{\circ}, M_{\infty}=1.8, R_{e}=10^{5}, \Delta \ell=10^{-3}$. 


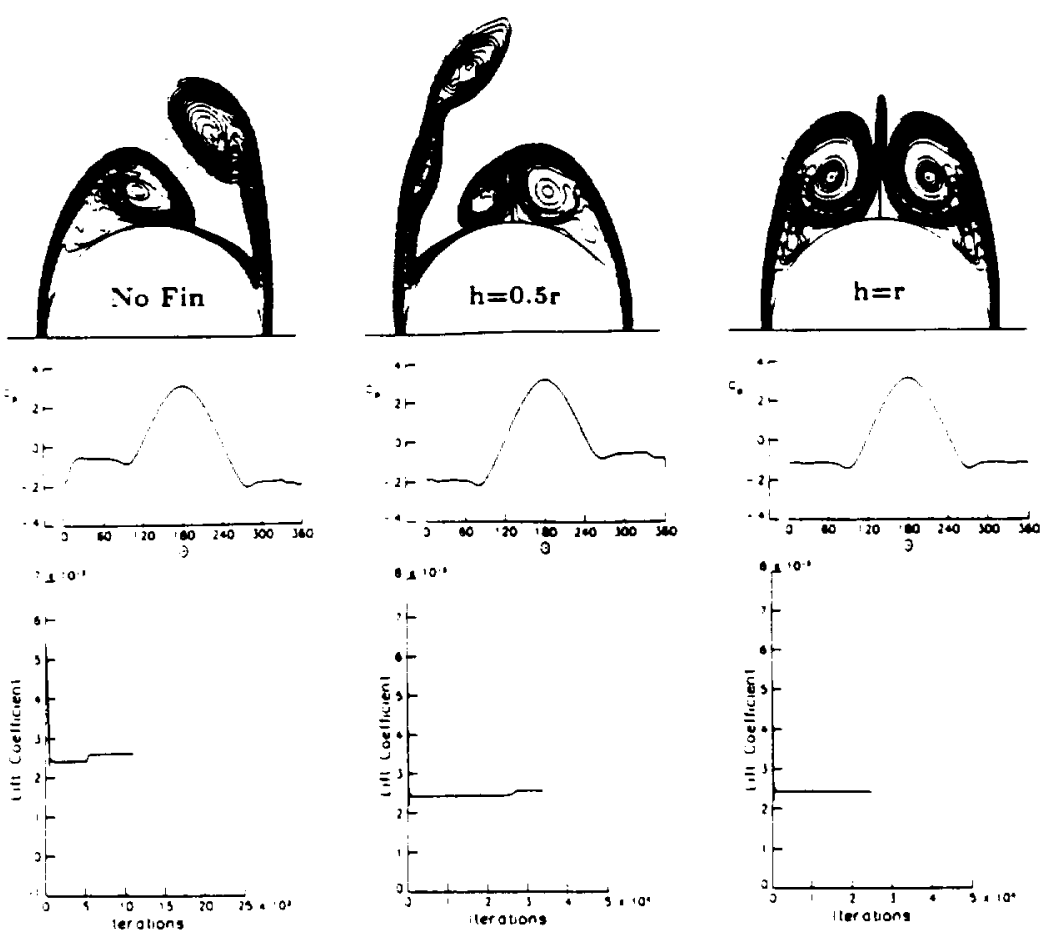

Figure 5. Passive control of asymmetric flow around a circular conc using a vertical fin. $\alpha=20^{\circ}, M_{\infty}=$ 1.8, $R_{e}=10^{5}, h=0.5 r$ and $r(r \equiv$ cone local radius).

$-$
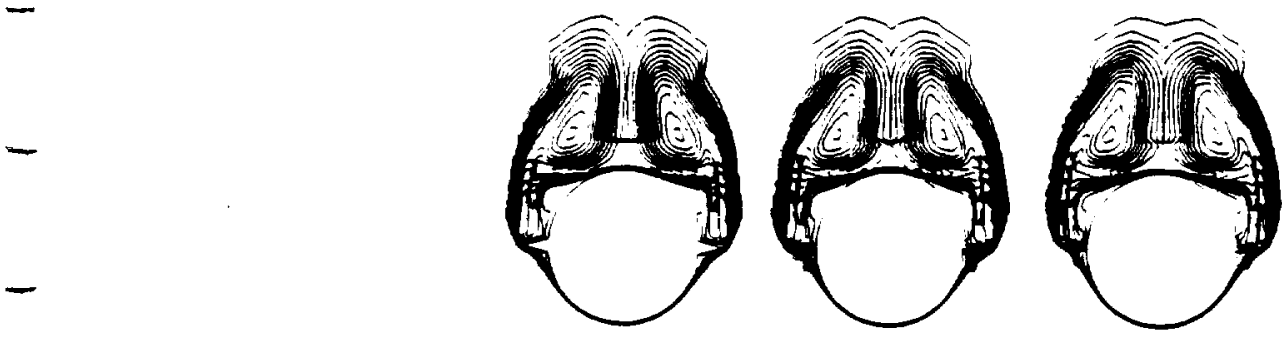

$\delta=0^{\circ}$
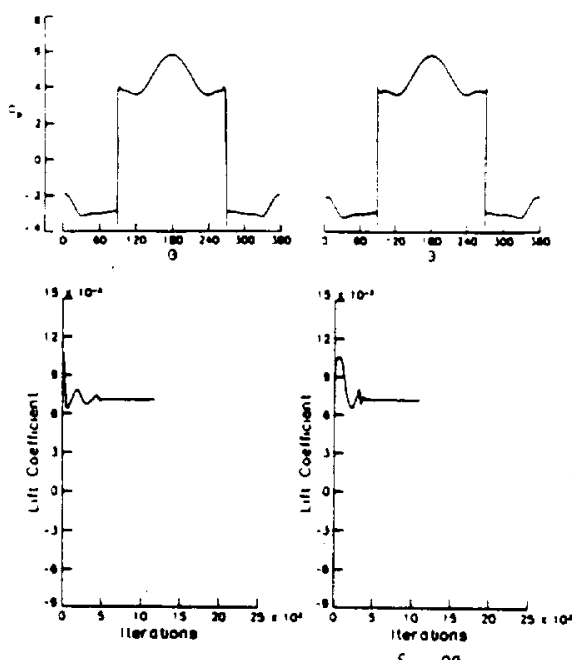

$\delta=0^{\circ}$ $\delta=10^{\circ}$

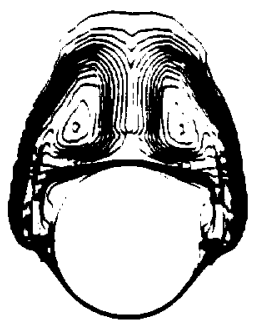

$\delta=-10^{\circ}$
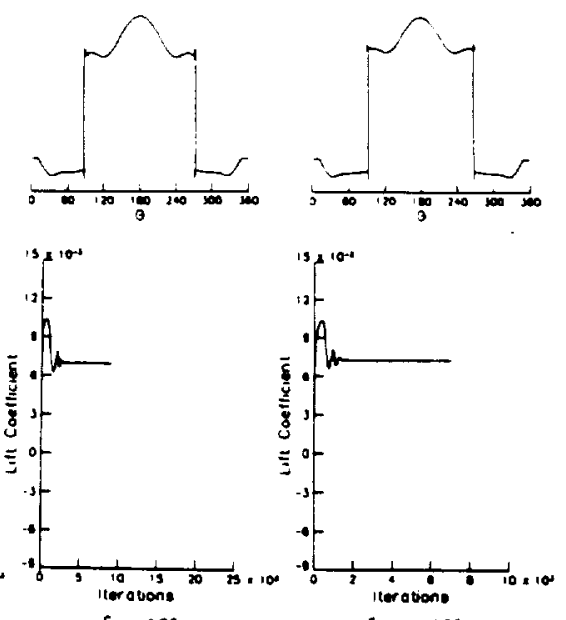

$\delta=-10^{\circ}$

Figure 6. Passive control of asymmetric flow around a circular cone using sharp-edged thick strakes and flat-plate strakes with different orientations. $\alpha=30^{\circ}, M_{\infty}=1.8, R_{e}=10^{5}, h=0.3 r$. 


$$
\begin{aligned}
& 20100 \\
& \text { pop }
\end{aligned}
$$

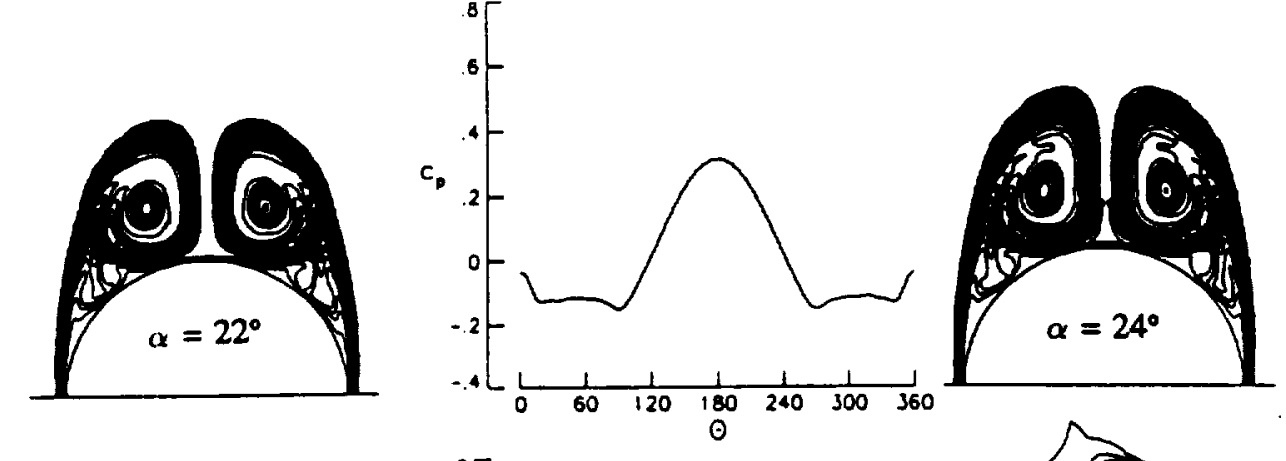

$$
\begin{aligned}
& 20 \text {. }
\end{aligned}
$$



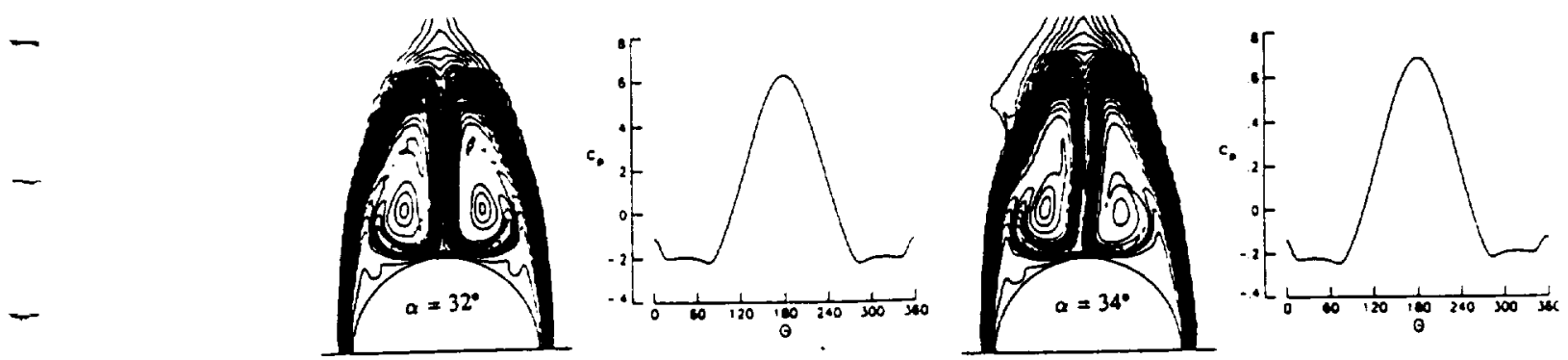

Heating

$-$
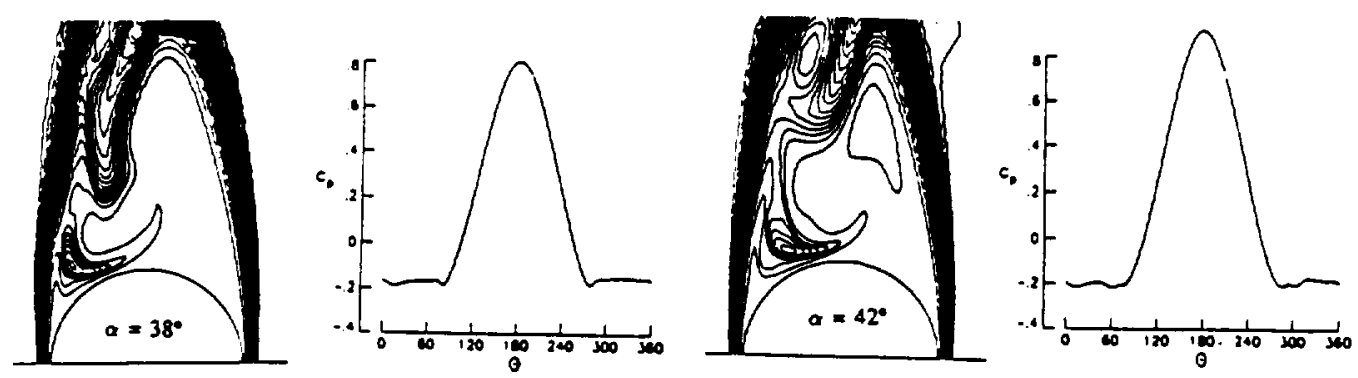

\section{Variable mass injection}

Figure 9. Hybrid heating-injection control: TPL contours and SP coefficient; $\alpha=20^{\circ}-42^{\circ}, M_{\infty}=1.8, R_{e}$ $=10^{5}, \dot{m}=0.05, T_{1}=5 T_{\infty}$ on whole surfacc.
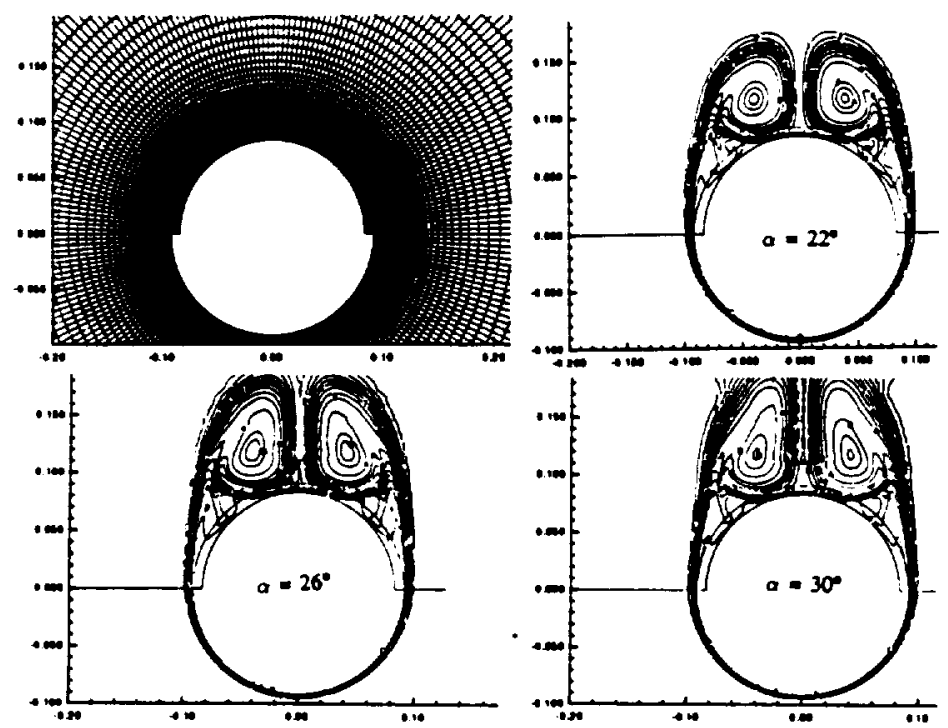

Figure 10. Typical multi-block grid and total pressure-loss contours for the tangential-flow injection control; $\alpha=22^{\circ}, 26^{\circ}, 30^{\circ} ; M_{\infty}=1.8, R_{e}=10^{5}$, parabolic velocity profile at lip exit, $\dot{m}_{\max }=0.2$. 
